\title{
Colonization in Piglets by Campylobacter Species and its Antimicrobial Profile
}

\author{
P. Muralikrishna ${ }^{1 *}$, B. Sunil ${ }^{1}$, Vrinda K. Menon ${ }^{1}$, Deepa Jolly ${ }^{1}$, C. Latha ${ }^{1}$, \\ Ashok Kumar ${ }^{2}$ and Safeer M. Saifudeen ${ }^{3}$ \\ ${ }^{1}$ Department of Veterinary Public Health, ${ }^{2}$ Indian Council of Agricultural Research, \\ ${ }^{3}$ University Goat and Sheep Farm, College of Veterinary and Animal Sciences, Mannuthy, \\ Thrissur, Kerala- 680651, India \\ *Corresponding author
}

\begin{tabular}{|l|}
\hline K e y w or d s \\
Campylobacteriosis, \\
Piglets, \\
Antimicrobial \\
resistance, \\
Prevalence, \\
Environment \\
\hline Article Info \\
\hline $\begin{array}{l}\text { Accepted: } \\
\text { 26 June } 2018 \\
\text { Available Online: } \\
\text { 10 July 2018 }\end{array}$ \\
\hline
\end{tabular}

\section{Introduction}

Campylobacter spp. is one of the major causes of foodborne bacterial infection and is among the most frequently reported zoonoses in humans worldwide with an estimated 400-500 million cases of diarrhoea each year (RuizPalacios, 2007). Campylobacter organisms are widespread in the environment and are present in the intestinal tracts of a wide range of birds 
and mammals, including domestic animals used for food production (Inglis et al., 2005). They are highly prevalent in food animals such as pigs, poultry, cattle, sheep and ostriches; and also in pet animals like cats and dogs. Pigs are considered as the main reservoir host for Campylobacter spp. and are capable of shedding these organisms asymptomatically in their faeces which can pose a threat to farm biosecurity. The wide range of reservoirs, contaminated environment and natural water presents an intricate situation (Humphrey et al., 2007). The colonization of the organism in piglets at the farm level occurs at an early age and pose a threat to farm biosecurity. Pigs can also carry a wide range of Campylobacter genotypes and piglets can acquire infection from mother and their associated environment which is contaminated (Weijtens et al., 1997). Antimicrobial agents are a leading weapon in the treatment of infectious diseases caused by bacteria and other microbes. The uncontrolled rise in resistant pathogens threatens lives and puts stress on the limited healthcare resources. The development and spread of resistance in zoonotic bacteria including Campylobacter with its reservoirs in healthy food producing animals and birds has become a global public health problem.

This study aims to provide an overall picture about colonization of Campylobacter spp. in piglets and influence of pigs, wild reservoirs and contaminating environment towards colonization in piglets. This investigation will also throw light on the antimicrobial susceptibility pattern of the isolates obtained during the study and assess the threat by resistant isolates towards farm biosecurity.

\section{Materials and Methods}

The present study was undertaken to analyse the colonisation of piglets by Campylobacter spp., occurrence of Campylobacter spp. in pigs, wild birds and environmental samples and associated threat towards farm biosecurity. The molecular confirmation of isolates, and antibiotic resistance profile of the positive isolates was also carried out.

\section{Collection of samples}

A total of 255 samples was collected from a government licensed farm in Thrissur, Kerala. Fifteen specific piglets from two litters were selected using the unique identification number. Rectal swabs were collected and examined at weekly intervals over the first 10 weeks of life. The sows and associated environment was also sampled on each visit. Samples consisted of piglet rectal swabs (zero to tenth week), sow and boar rectal swabs, faecal samples from pigs, wild bird's droppings, dog rectal swabs, human faecal samples, feed, water, water from wallowing tank, soil, hand swabs and foot swabs. Swab samples from the animals and humans were collected using sterile cotton swabs (HiMedia, India) dipped in Cary Blair medium with charcoal. Faecal samples from pigs and humans were collected in sterile bottles. Feed, drinking water samples, wallowing tank water samples and soil were collected in sterile bottles. The aseptically collected samples were immediately transported at $4^{\circ} \mathrm{C}$ to the laboratory and were processed within four hours of collection to ensure that the organisms remain viable and culturable.

\section{Processing of samples}

All the samples collected were subjected to isolation and identification of Campylobacter spp as per the procedure described by OIE terrestrial manual, 2017 with modifications. The selective enrichment of the samples was carried out in Blood Free Campylobacter (modified Charcoal Cefoperazone Deoxycholate, mCCD) broth (HiMedia, India) under microaerophilic conditions at $42^{\circ} \mathrm{C}$ for $48 \mathrm{~h}$. All the samples except rectal swabs were 
subjected to enrichment at the ratio of 1:9 in mCCD broth. Rectal swab samples were swabbed directly onto P-mCCDA (Polymyxin B supplemented modified Charcoal Cefoperazone Deoxycholate Agar) plates and incubated under microaerophilic conditions at $42^{\circ} \mathrm{C}$ (Thermo $\mathrm{CO}_{2}$ incubator) for $48 \mathrm{~h}$.

\section{Molecular detection}

All the positive isolates were subjected to multiplex polymerase chain reaction (mPCR) for the confirmation of Campylobacter spp. All the isolates were analysed for the presence of Campylobacter genus specific 16SrRNA gene (816 bp). Species level identification was done by the detection of $C$. jejuni specific mapA gene (589 bp) and C. coli specific ceuE gene (489 bp). All the Campylobacter isolates were analysed for the presence of $c a d \mathrm{~F}$, a conserved virulence gene (400 bp) (Fig. 1 and 2).

\section{Antibiogram}

All Campylobacter isolates were examined for their antimicrobial drug susceptibility/resistance pattern by disc diffusion method, as per the procedure described by Baserisalehi et al., (2007) against 33 different antibiotics.

\section{Results and Discussion}

On analysing 255 samples collected from various sources which included rectal swabs from piglets, pigs and dogs, faecal samples from pigs and humans, wild birds droppings, environmental and feed samples and worker's hand swabs and foot swabs 84 samples were positive for Campylobacter spp.

Sampling of piglets was initiated for piglets three days after birth where sows were found to carry Campylobacter spp. but none of the piglets were positive for Campylobacter spp. Out of 15 piglets screened regularly at weekly interval, five piglets started excreting Campylobacter spp. from fourth week onwards with a regular pattern of shedding among piglets. By the end of tenth week, ten piglets were positive for Campylobacter spp. with a prevalence rate of 66.66 per cent. Till six weeks, piglets were excreting Campylobacter jejuni and some had mixed infection of $C$. coli and $C$. jejuni which was confirmed by molecular techniques and piglets were weaned and kept in boar pen. Boar rectal swabs were also positive for Campylobacter spp. From seventh week $C$. coli was predominantly isolated from piglets. By the end of tenth week all piglets started excreting C. coli. Overall Campylobacter coli (37/54) was the predominant species isolated followed C. jejuni (17/54).

On screening of pig rectal swabs and faecal samples, prevalence rate of 45 per cent and 30 per cent was obtained with a higher prevalence among sows. Thirty per cent prevalence was recorded from wild birds mainly from Corvus splendens. Two feed samples were positive for Campylobacter spp. which was obtained from pens of pigs fed with chicken waste. Three wallowing water samples collected from pregnant sow and adult pens were positive for Campylobacter spp. Two soil samples obtained from the vicinity of sheds were contaminated with Campylobacter spp. and 40 per cent of worker's foot swab samples collected after operations were positive for Campylobacter spp. Of three dog rectal swab samples collected from the farm, one sample was found to be positive for Campylobacter spp. (Table 1).

\section{Antibiotic resistance profiling}

All the positive isolates of Campylobacter spp. obtained from samples were subjected to antibiotic sensitivity test by standard disc diffusion method. 
Table.1 Occurrence of Campylobacter spp. in government licenced farm in Thrissur

\begin{tabular}{|c|c|c|c|c|}
\hline Sl.no & Source & $\begin{array}{c}\text { Total no. of } \\
\text { samples }\end{array}$ & $\begin{array}{c}\text { Positive } \\
\text { samples }\end{array}$ & $\begin{array}{c}\text { Occurrence } \\
\%\end{array}$ \\
\hline $\mathbf{1}$ & Rectal swabs - Piglets (0-10 weeks) & 150 & 54 & 36 \\
\hline $\mathbf{2}$ & Pigs- Rectal swabs & 20 & 9 & 45 \\
\hline $\mathbf{3}$ & Pigs - Faecal samples & 20 & 6 & 30 \\
\hline $\mathbf{4}$ & Wild birds- droppings & 10 & 3 & 30 \\
\hline $\mathbf{5}$ & Feed & 10 & 2 & 20 \\
\hline $\mathbf{6}$ & Drinking water & 10 & - & - \\
\hline $\mathbf{7}$ & Wallowing tank samples & 10 & 3 & 30 \\
\hline $\mathbf{8}$ & Soil & 10 & 2 & 20 \\
\hline $\mathbf{9}$ & Worker's hand swabs and foot & 10 & 4 & 40 \\
\hline $\mathbf{1 0}$ & swabs & 2 & - & - \\
\hline $\mathbf{1 1}$ & Faecal sample - Workers & 3 & 1 & 33.33 \\
\hline & Dog rectal swabs & $\mathbf{2 5 5}$ & $\mathbf{8 4}$ & $\mathbf{3 2 . 9}$ \\
\hline
\end{tabular}

Fig. 1 Multiplex PCR for Campylobacter jejuni

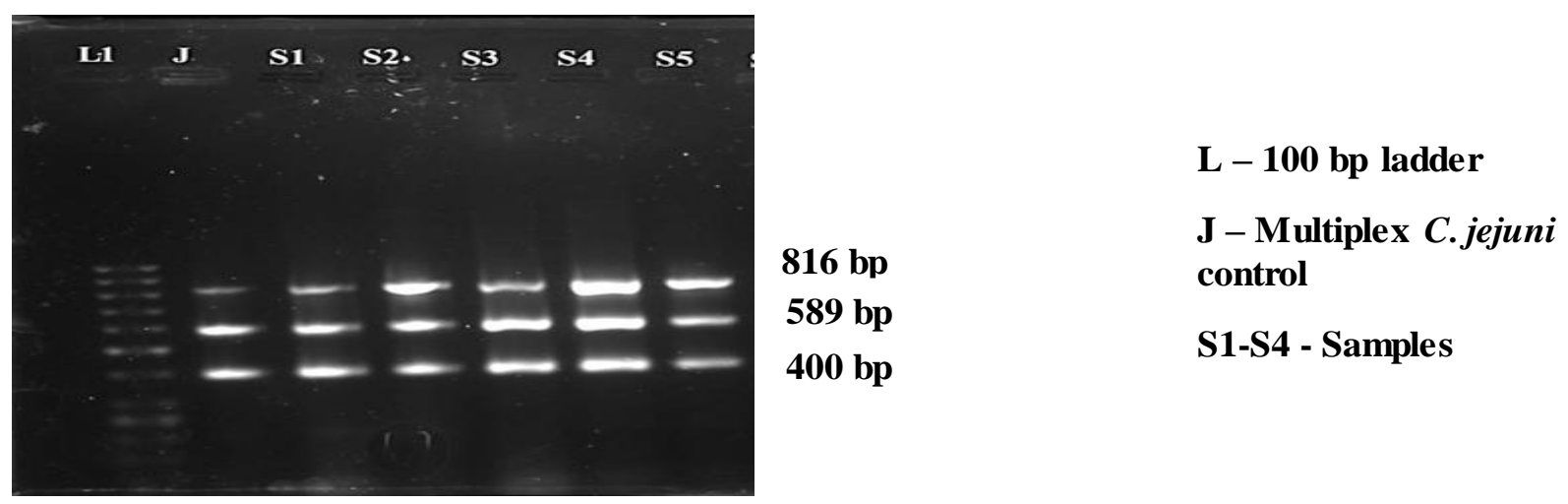

Fig.2 Multiplex PCR for Campylobacter coli

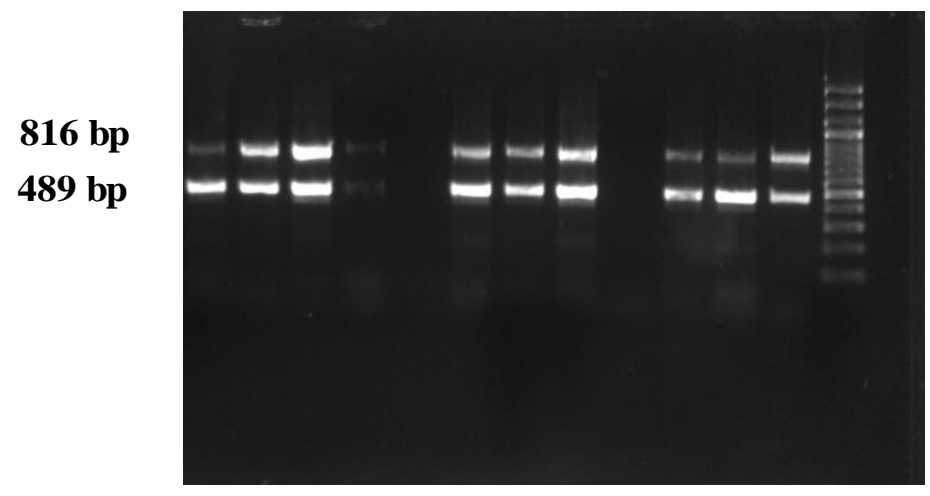

L - 100 bp ladder

$\mathrm{L}_{1}$ - Multiplex C. coli control

$\mathbf{S}_{2}-\mathbf{S}_{11}$ - Samples 
The prevalence of Campylobacteriosis has been reported worldwide and it is one of the most widespread zoonotic gastro enteric infectious diseases of the last century. Pathogenicity of Campylobacter spp. is mainly attributed by its low infectious dose, toxin production, carrier status in poultry and coccoid dormant stage. These organisms have become the major pathogens of foodborne illnesses because of its severe consequences of infection like GuillainBarre syndrome, reactive arthritis and irritable bowel syndrome. In this study, carriage rate among piglets was about 33.33 per cent from fourth week of age, which was lower than that reported by Weijtens et al., (1997), who found 85 per cent of piglets positive after four weeks. Steinhauserova et al., (2001) found 41 per cent of healthy piglets up to 8-week-old positive which was mostly similar to results in this study. Piglets started excreting Campylobacter spp. from the fourth week onwards with a regular pattern of shedding. By the end of tenth week, ten piglets were positive for Campylobacter spp. with a prevalence rate of 66.66 per cent. Regular increase in incidence among piglets was mainly from sows which carried the infection which points to be a likely source of infection. Comparable results were obtained from the study conducted by Weijetens et al., (1997), who found out that 9 out of 10 sows can carried Campylobacter spp. and could infect 85 per cent of piglets with during initial four weeks of life. Alter et al., (2005) also found similar results where they found incidence of 56.6 per cent at fourth week and genotypic similarities was found between sow and piglets. Other probable sources can be from the boar which was positive and piglets were shifted into its pen after weaning as well as from piglets which was positive initially. However, Thakur and Gebreyes (2005) found that 77 per cent of nursery pigs carried $C$. coli on antibiotic free farms but on farms using antibiotics the prevalence was only 28 per cent. Hence farming practices can directly affect $C$. coli prevalence among piglets.

All the piglets excreted $C$. jejuni till seven weeks which can be mainly be attributed to feeding of chicken waste which was positive for Campylobacter spp. and presence of wild birds which may have contributed to $C$. jejuni contamination. Similar results were obtained from the work done by Jensen et al., (2006). They observed that 35 per cent of environmental samples (soil and water) were positive for Campylobacter spp. and wildlife reservoirs like rats, crow-birds also contributed predominantly to paddock contamination predominantly with $C$. jejuni. Ramonaite et al., (2015) also observed higher prevalence of $C$. jejuni among crows (39.2 per cent) which contribute to paddock contamination. From seventh week, $C$. coli was predominantly isolated from piglets and by the end of tenth week all piglets started excreting $C$. coli which can be mainly be attributed to alteration of intestinal colonisation by $C$. coli and contributed by carrier sows and boar which contaminate paddock environment and also from contaminated soil and water. Trimble et al., (2013) analyzed soil, compost and processing waste water and found a prevalence rate of 64.3 per cent in soil and 45 per cent in waste water and these contributed to paddock contamination. Workers also contributed to contamination of associated pens as 40 per cent of samples analyzed after operations were positive for Campylobacter coli.

All the $20 C$. jejuni isolates obtained from different sources were subjected to antibiotic sensitivity test by standard disk diffusion method. Campylobacter jejuni isolates showed more than 90 per cent sensitivity to clindamycin and nitrofurantoin which is in tune with the findings of Joby (2016) from Thrissur. Amikacin, kanamycin, streptomycin, chloramphenicol, ceftazidime and levofloxacin sensitivity patterns is in agreement with the findings of Joby (2016). In this resistance exhibited by the isolates against azithromycin and erythromycin was 97.37 and 92.10 per cent respectively which was in tune with results obtained by Karikari et al., (2017) from Africa. All the $64 C$. coli isolates obtained from samples were sensitive to nitrofurantoin which is in accordance with the findings of Joby 
(2016). The sensitivity patterns of amoxyclav (62.50 per cent) and levofloxacin (20.83 per cent) are in accordance with Dadi and Asrat (2008). Parkar et al., (2013) showed similar results for ampicillin (62.50 per cent) and gentamycin (sensitivity patterns as found in the present study. Hundred per cent of the isolates were resistant to azithromycin, ceftazidime, cotrimoxazole and ofloxacin which are in agreement with the reports of Baserisalehi et al., (2007). The resistance of fluoroquinolone group (ciprofloxacin and levofloxacin) and the resistance patterns for clindamycin, erythromycin and gentamicin of the present study are in agreement with the findings of Han et al., (2016).

Pigs and piglets have been reported as carriers of several genotypes of Campylobacter spp. The present study conforms to this finding and shows that piglets are probably infected from their mother and the associated environment. Feeding of chicken waste and wild birds contaminating the paddock environment with $C$. jejuni can act as a source of contamination and pigs can readily acquire $C$. coli from their surroundings, in turn leading to permanent colonization in the intestines of piglets. The dominant species observed in the piglets studied changed constantly over the 10 -week sampling period, but the trend was for $C$. coli to replace the $C$. jejuni which showed predominance in the beginning. In addition, the sow can acquire new species from her piglets and surroundings, and also display a constant change in the dominant genotypes detected.

In conclusion, Campylobacter spp. are most widely distributed and found mostly in warm blooded animals. The study revealed weekly prevalence of Campylobacter spp. in piglets and sources which contributed to colonisation of organism in piglets. As food safety and its association with biosecurity are highly significant, the potential threat to farm biosecurity due to colonisation in piglets is significantly higher. Contamination of paddock environment can contribute to transmission of Campylobacter spp. among animals and occupational group. In order to reduce the risk of contamination of food and infections caused by Campylobacter spp., it is important to adopt appropriate practices for reducing Campylobacter colonization in pigs and hygienic practices throughout the swine production chain. The pathogenic potential of the organism is evident due to the presence of the virulence gene of Campylobacter spp. The development of resistance to more antibiotics can be of public health significance. Development of strict biosecurity measures should be adopted in the farm in order to reduce environmental contamination and transmission. An antimicrobial intervention is necessary to reduce Campylobacter spp. in colonized pigs and piglets.

\section{Acknowledgement}

Financial assistance provided by Indian Council of Agricultural Research (ICAR) through Outreach Programme on Zoonotic Diseases (OPZD) for the research work is gratefully acknowledged.

\section{References}

Alter, T., Gaull, F., Kasimir, S., Gürtler, M., Mielke, H., Linnebur, M. and Fehlhaber, K., 2005. Prevalences and transmission routes of Campylobacter spp. strains within multiple pig farms. Vet. Microbiol. 108:251-261.

Baserisalehi, M., Bahador, N. and Kapadnis, B.A. 2007. A comparison study on antimicrobial susceptibility of Campylobacter spp. isolates from faecal samples of domestic animals and poultry in India and Iran. J. Biol. Sci. 7: 977-980

Chon, J.W., Hyeon, J.Y., Yim, J.H., Kim, J.H., Song, K.Y. and Seo, K.H. 2012. Improvement of modified charcoal cefoperazone deoxycholate agar by supplementation with a high concentration of polymyxin B for detection of Campylobacter jejuni and $C$. coli in chicken carcass rinses. Appl. Environ. Microbiol. 78: 1624-1626. 
Dadi, L. and Asrat, D. 2008. Prevalence and antimicrobial susceptibility profile of thermotolerant Campylobacter strains in retail raw meat products in Ethiopia. Ethiop. J. Hlth. Dev. 22: 195-200.

EFSA. 2008. Foodborne antimicrobial resistance as a biological hazard. Scientiûc opinion of the panel on biological hazards. EFSA J. 765: 1-87.

EUCAST. 2017. European Committee on Antimicrobial Susceptibility Testing. Breakpoint tables for interpretation of MICs and zone diameters. Version 7.1. EUCAST; 2017.

Han, X., Zhu, D., Lai, H., Zeng, H., Zhou, K., Zou, L., Wu, C., Han, G. and Liu, S. 2016. Prevalence, antimicrobial resistance profiling and genetic diversity of Campylobacter jejuni and Campylobacter coli isolated from broilers at slaughter in China. Food Control. 69: 160-170.

Humphrey, T., O’Brien, S., Madsen, M. 2007. Campylobacters as zoonotic pathogens: a food production perspective. Int. J. Food. Microbiol. 117: 237-57.

Inglis, G. D., McAllister, T. A., Busz, H. W., Yanke, L. J., Morck, D. W., Olson, M. E., and Read, R. R. 2005. Effects of sub therapeutic administration of antimicrobial agents to beef cattle on the prevalence of antimicrobial resistance in Campylobacter jejuni and Campylobacter hyointestinalis. Appl. Environ. Microbiol. 71(7): 3872-3881.

Jensen, A.N., Dalsgaard, A., Baggesen, D.L. and Nielsen, E.M., 2006. The occurrence and characterization of Campylobacter jejuni and $C$. coli in organic pigs and their outdoor environment. Vet. Microbiol. 116: 96-105.

Joby, E.J. 2016. Occurrence of Campylobacter spp. in chicken egg production chain. M.V.Sc thesis, Kerala Veterinary and Animal Sciences University, Pookode,
99 p.

Karikari, A.B., Obiri-Danso, K., Frimpong, E.H. and Krogfelt, K.A. 2017. Multidrug resistant Campylobacter in faecal and carcasses of commercially produced poultry. Afr. J. Microbiol. Res. 11: 271277.

Parkar, S.F., Sachdev, D., Kamble, A., Suresh, G., Munot, H., Hanagal, D., Shouche, Y. and Kapadnis, B. 2013. Prevalence, seasonality and antibiotic susceptibility of thermophilic Campylobacters in ceca and carcasses of poultry birds in the live-bird market. Afr. J. Microbiol. Res. 7: 24422453.

Ramonaitè, S., Novoslavskij, A., Zakarienè, G., Aksomaitienè, J. and Malakauskas, M., 2015. High prevalence and genetic diversity of Campylobacter jejuni in wild crows and pigeons. Curr. Microbial. 71(5): 559-565.

Ruiz-Palacios, G. M. 2007. The health burden of Campylobacter infection and the impact of antimicrobial resistance: playing chicken. Clin. Infect. Dis. 44(5): 701-703.

Thakur, S. and Gebreyes, W.A., 2005. Campylobacter coli in swine production: antimicrobial resistance mechanisms and molecular epidemiology. J. Clin. Microbiol. 43(11): 5705-5714.

Trimble, L.M., Alali, W.Q., Gibson, K.E., Ricke, S.C., Crandall, P., Jaroni, D., Berrang, M. and Habteselassie, M.Y. 2013. Prevalence and concentration of Salmonella and Campylobacter in the processing environment of small-scale pastured broiler farms. Poult. Sci. 92: 3060-3066.

Weijtens, M.J.B., der Plas, J., Bijker, P.G.H., Urlings, H.A.P., Koster, D., van Logtestijn, J.G. and Huis in't Veld, J.H.J. (1997) The transmission of campylobacter in piggeries: An epidemiological study. $J$. Appl. Micro. 83: 693-698.

\section{How to cite this article:}

Muralikrishna, P., B. Sunil, Vrinda K. Menon, Deepa Jolly, C. Latha, Ashok Kumar and Safeer M. Saifudeen. 2018. Colonization in Piglets by Campylobacter Species and its Antimicrobial Profile. Int.J.Curr.Microbiol.App.Sci. 7(07): 3801-3807.

doi: https://doi.org/10.20546/ijcmas.2018.707.441 